\title{
Correction to: Chitosan-based hydrogels to treat hydrofluoric acid burns and prevent infection
}

\author{
Shu-Wei Huang ${ }^{1} \cdot$ Fang-Ching Yeh ${ }^{2} \cdot$ You-Ren $\mathrm{Ji}^{3} \cdot$ Ying-Fu Su${ }^{4} \cdot$ Ying-shih $\mathrm{Su}^{5,6} \cdot \mathrm{Ming}$-Hsien Chiang ${ }^{7}$. \\ Shian-Chiuan Tzeng ${ }^{8} \cdot$ Chien-Yao Fu ${ }^{9} \cdot$ Aristine Cheng $^{10,11} \cdot$ Yung-Chih Wang $^{12} \cdot$ Yi-Tzu Lee $^{1,13}$
}

Published online: 10 December 2021

(c) Controlled Release Society 2021

\section{Correction to: Drug Delivery and Translational Research (2021) 11:1532-1544 https://doi.org/10.1007/s13346-021-01007-3}

Yung-Chih Wang's given name is correct as reflected here.

The article note Yi-Tzu Lee and Yung-Chih Wang contributed equally to this work was missing from the original online version of this article.

The original article was corrected.

Publisher's Note Springer Nature remains neutral with regard to jurisdictional claims in published maps and institutional affiliations.

The original article can be found online at https://doi.org/10.1007/ s13346-021-01007-3.

Ying-Fu Su

Yi-Tzu Lee

s851009@yahoo.com.tw

1 Department of Emergency Medicine, Taipei Veterans General Hospital, 11217 Taipei, Taiwan

2 Division of Infectious Diseases, Department of Internal Medicine, Taichung Armed Forces General Hospital, Taichung 40466, Taiwan

3 Department of Biomedical Engineering, National Taiwan University, Taipei 10023, Taiwan

4 Department of Emergency, Kuang Tien General Hospital, Taichung 43303, Taiwan

5 Division of Infectious Diseases, Department of Internal Medicine, Wan Fang Medical Center, Taipei Medical University, Taipei 11608, Taiwan

6 Department of Internal Medicine, School of Medicine, College of Medicine, Taipei Medical University,

Taipei 10031, Taiwan
Department and Graduate Institute of Biology and Anatomy, National Defense Medical Center, Taipei 11490, Taiwan

8 Department of Biomedical Engineering, National Taiwan University, Taipei 10617, Taiwan

9 Division of Orthopedics, Taichung Armed Forces General Hospital, Taichung 40466, Taiwan

10 Department of Internal Medicine, National Taiwan University Hospital, Taipei, Taiwan

11 College of Medicine, National Taiwan University, Taipei, Taiwan

12 Division of Infectious Diseases and Tropical Medicine, Department of Internal Medicine, Tri-Service General Hospital, National Defense Medical Center, Taipei, Taiwan

13 Faculty of Medicine, School of Medicine, National Yang-Ming University, Taipei, Taiwan 